\title{
A Novel Fingerprint Matching Method Combining Geometric and Texture Features
}

\author{
Mei Xie, Chengpu Yu and Jin Qi \\ University of Electronic Science and Technology of China. \\ Chengdu,P.R.China Post Code:610054 \\ xiemei@ee.uestc.edu.cn
}

\begin{abstract}
In this paper, we proposed a new fingerprint matching algorithm based on local geometric feature of fingerprint minutia and texture feature for each minutia. To describe the geometric feature of fingerprint minutia, we build a bi-minutia based bar model and get the geometric relationship between the bar and ridges of two candidate minutia; to demonstrate the texture feature, we creatively adopt gradient angular histogram in the neighborhood region of minutia. Meanwhile, changeable sized boundary box of unique area adopted for minutia matching make this algorithm more robust to nonlinear fingerprint deformation. Finally, experimental results on the database FVC2004 demonstrate that our method is effective and reliable, whilst the matching accuracy can be improved to some extent after using gradient angular histogram as texture feature without adding extra amount of calculation.
\end{abstract}

\section{Introduction}

Automatic fingerprint recognition, which is established in modern information technology, is wildly used to civilian purposes such as access control, financial security, and so on. Fingerprint recognition technology is based on the reality that fingerprint of each person have its uniqueness and unchangeable properties.

Many researchers have made progress in the fingerprint matching algorithms. These algorithms can mainly be divided into several categories as follows: (1) minutia and ridge based method [15]; (2) minutia and texture based method [14]; (3) graph based method [12] [13]; (4) tri-minutia structure based method [7]. All the conspicuous features used in above methods are related and complemented. Taking into account of advantages of above methods, we propose the method based on bi-minutia based bar model, and add texture feature to fingerprint representation to improve the matching efficiency and accurateness.

The algorithm introduced in this paper adopts local geometric feature of mi- 
nutia and texture feature around minutia. Bi-minutia based bar model used in this method can compromise the deficiency of solo-minutia based and tri-minutia based model, and it can effectively and reliably extract matched minutia pairs. Meanwhile, the gradient angular histogram as texture feature can generally reflect the profile of fingerprint ridge edges and the minutia type (bifurcation or ending).

This paper is organized as follows. Section 2 briefly describes preprocessing of fingerprint image. Section 3 demonstrates local structure and reference minutia selection. Section 4 illustrates fingerprint alignment and global matching. Section 5 shows the experimental results on the database FVC2004. In the end, we draw the conclusion in section 6 .

\section{Preprocessing of fingerprint image}

In this paper, we adopt fingerprint features not only from thinned ridge image, such as minutia position and geometric feature, but also from original gray image, such as gradient angular histogram as texture feature. Since low quality fingerprint image often contains noises and contamination, it requires us to preprocess the image to enhance image. Main steps involved in the preprocessing include fingerprint segmentation, block orientation estimation, image enhancement, image binarization, thinning, and minutia extraction. LinHong [1] introduces the orientation estimation method based on gradient vectors of fingerprint ridges, which could compute directions more accurately in low quality image, see Figure 1(b). Zhu [2] proposes Gabor filtering enhancement method can overcome the deficiency that occurs when using method in LiHong[1], see Figure 1(c). X.P.Luo[3] describes binarization and post processing of fingerprint image with method based on knowledge and method based on combination of statistic and structure, see Figure 1(d).

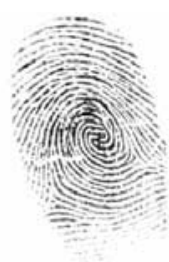

(a)

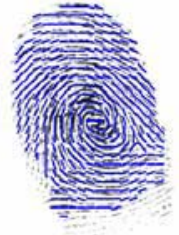

(b)

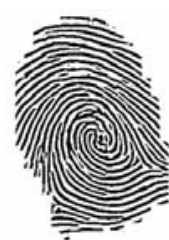

(c)

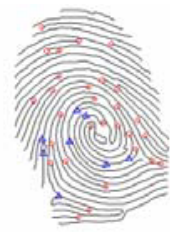

(d)

Fig. 1 Fingerprint preprocessing.(a)An original fingerprint in DB1_Aof FVC2004,(b)its block orientation field,(c)enhanced image,(d) thinned image of fingerprint image

\section{Local structure description and reference minutia selection}

Minutia features (ending or bifurcation) are salient and stable features for fin- 
gerprint image of different discrimination. However, external interference will cause many pseudo minutias and create large error, and using multi-minutia based model can resist interference to some extent.

\subsection{Feature description of solo-minutia based model and calculation of gradient angular histogram}

Vector set of minutia $M^{F}=\left\{M_{i}^{F}=\left(x_{i}^{F}, y_{i}^{F}, \alpha_{i}^{F}, h_{i 1}^{F} \Lambda h_{i 8}^{F}\right) ;\left|M^{F}\right| \geq i \geq 1\right\}$ denote all comprehensive minutia in the fingerprint. Where $\left|M^{F}\right|$ is the number of minutia in a fingerprint image. $M_{i}^{F}$, the $i$ th minutia, is denoted by a feature vector $x_{i}^{F}, y_{i}^{F}, \alpha_{i}^{F}, h_{i 1}^{F} \Lambda h_{i 8}^{F}$. Where

(a) $x_{i}^{F}, y_{i}^{F}$ denotes its coordinates;

(b) $\alpha_{i}^{F}$ denotes the tangent direction of the ridge where the minutia locates;

(c) $h_{i 1}^{F} \Lambda h_{i 8}^{F}$ denotes gradient angular histogram in the circular neighborhood region whose radius is $r$. The algorithm of gradient vector computation [5] has been described in the section of fingerprint orientation field estimation. Then we transform the vector in Euclidean space to the polar coordinates, thus the magnitude and direction can be calculated as follows:

$$
\begin{gathered}
\nabla f=\operatorname{mag}(\nabla f)=\left[G_{x}^{2}+G_{y}^{2}\right]^{1 / 2} \\
\alpha=\arctan \left(\frac{G_{y}}{G_{x}}\right)
\end{gathered}
$$

$\operatorname{mag}(\bullet)$ and $\nabla f$ in formula (6) represent magnitude calculation operator and magnitude value respectively. $\alpha$ in formula (7) represents direction of gradient vector, which is perpendicular to the direction of the corresponding image edge.

Gradient angular histogram is considered as texture feature for fingerprint recognition. Utilization of gradient angular histogram in this paper has much preponderance which are showed as follows: 1) it is more stable to the change of illumination, because it extracts the gradient angles of larger gradient magnitude here; 2) it is invariant to scale and displacement of the image; 3) it is invariant to rotation. Here, we define the tangent angle of the ridge where minutia locates as the reference direction.
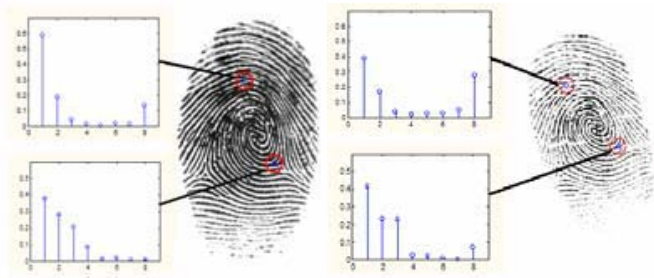

Fig. 2 Gradient angular histograms of two matched minutia pairs from the same fingerprint illustrated as stem graphs. (1) Upper stem graphs represent gradient angular histogram of endings, similarity between them is 0.929 ; (2) Lower stem graphs illustrate that of bifurcations, similarity between them is 0.975 . 
Gradient angular histograms of two matched minutia pairs have been demonstrated in Figure 2. In the stem graph, direction 1 and direction 8 are neighborhood. From the gradient angular histogram, we can observe that all the gradient angles concentrate in the region whose center is the direction of ridge where minutia locates. Gradient angular histogram adopted here can reflect the information of minutia type to some extent, and it also can reflect the texture information around the minutia.

\subsection{Feature description of bi-minutia based model}

Vector set of bi-minutia based model $E^{F}=\left\{E_{i}^{F}=\left(p_{i}^{F}, q_{i}^{F}, l_{i}^{F}, c_{i}^{F}, \theta_{i}^{F}, u_{i}^{F}, v_{i}^{F}\right),\left|E^{F}\right| \geq i \geq 1\right\}$ represents information of all bi-minutia bars, and these features are showed in Figure 3.

(a). $\left|E^{F}\right|$ is the size of bi-minutia bar set.

(b). $p_{i}^{F}, q_{i}^{F}$ denote the serial numbers in the minutia set $M^{F} \cdot M_{p_{i}}^{F}$ and $M_{q_{i}}^{F}$ are two ending minutia of bi-minutia bar $E_{i}^{F}$, and they contain all features listed in the minutia set.

(c). $l_{i}^{F}=\left\|\left(x_{p_{i}}^{F}, y_{p_{i}}^{F}\right)-\left(x_{q_{i}}^{F}, y_{q_{i}}^{F}\right)\right\|$ denotes the length of bi-minutia bar, and $\left(x_{p_{i}}^{F}, y_{p_{i}}^{F}\right),\left(x_{q_{i}}^{F}, y_{q_{i}}^{F}\right)$ represent coordinates of $M_{p_{i}}^{F}$ and $M_{q_{i}}^{F}$ respectively. In order to limit the number of bi-minutia bars in a fingerprint image, the length of bi-minutia bars are confined in range $L_{l} \leq l_{i}^{F} \leq L_{h}$

(d). $c_{i}^{F}$ denote the number of fingerprint ridges that bar $E_{i}^{F}$ passes through, because there will exist large error if we only calculate Euclidean distance; however, combining $c_{i}^{F}$ to Euclidean distance can accurately describe corresponding distance.

(e). $\theta_{i}^{F}=\arctan \left(\begin{array}{c}x_{p_{i}}^{F}-x_{q_{i}}^{F} \\ y_{p_{i}}^{F}-y_{q_{i}}^{F}\end{array}\right)$ denotes the direction of bi-minutia bar.

(f). $u_{i}^{F}=\min \left\{\left|\alpha_{p_{i}}^{F}-\theta_{p_{i}}^{F}\right|, \pi-\left|\alpha_{p_{i}}^{F}-\theta_{p_{i}}^{F}\right|\right\}, v_{i}^{F}=\min \left\{\left|\alpha_{q_{i}}^{F}-\theta_{q_{i}}^{F}\right|, \pi-\left|\alpha_{q_{i}}^{F}-\theta_{q_{i}}^{F}\right|\right\}$ denote bi-minutia bar's directional deviations from $\alpha_{p_{i}}^{F}$ and $\alpha_{q_{i}}^{F}$, and this feature have rotation invariant ability..

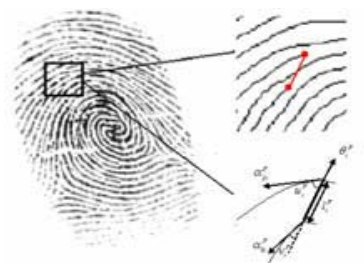

Fig. 3 Demonstration of minutia structure and features in a ridge image 
Influenced by the low quality in a fingerprint, minutia extracted might not be so stable, hence we haven't added minutia type as a character in bi-minutia based model [11]. Here, the angular histogram around the minutia as the texture can express the type of minutia.

\subsection{Selection of reference minutia pairs for fingerprint matching}

As fingerprint image are extracted in the same device, subtle deformation of the fingerprint can be omitted. Solo-minutia based model [6] used in fingerprint matching will generate lots of pseudo matched minutia pairs; tri-minutia based model [7] used in fingerprint matching might have difficulty in looking for entirely matched triangle composed of three minutia, so that we adopt bi-minutia bar based model as the compromise of two above models.

Suppose bi-minutia bar sets $E^{F}$ and $E^{G}$ denote fingerprint representation of input and template fingerprints, and matching criteria includes:

(a). Constraint of bar-length: $\left|l_{i}^{F}-l_{j}^{G}\right| \leq d_{0}$, where $d_{0}$ usually makes value about half the ridge period in the fingerprint;

(b). Constraint of ridge numbers the bar passes through: $\left|c_{i}^{F}-c_{j}^{G}\right| \leq n_{0}$, where $n_{0}$ usually makes value about 1 or 2 ;

(c). Constraint of directional deviation of ridges and bi-minutia bar: $\left|u_{i}^{F}-u_{j}^{G}\right| \leq u_{0}$ and $\left|v_{i}^{F}-v_{j}^{G}\right| \leq v_{0}$, where $u_{0}$ and $v_{0}$ usually make values lower than $\pi / 12$.

(d). Constraint of texture similarity: $s\left(M_{p_{i}}^{F}, M_{p_{j}}^{G}\right) \geq s_{0}, s\left(M_{q_{i}}^{F}, M_{q_{j}}^{G}\right) \geq s_{0}$, where $s_{0}$ should be larger than 0.9 , which is obtained from numerous experiment. The similarity of two vector $s\left(h_{i}, h_{j}\right)$ can be estimated as correlation coefficient:

$$
\left.\underset{s\left(h_{i}, h_{j}\right.}{\rho}\right)=\frac{\left\langle h_{i}, h_{j}>\right.}{\left\|h_{i}\right\|\left\|_{j}\right\|}=\frac{\sum_{k=1}^{L} h_{i}(k) h_{j}(k)}{\sqrt{\sum_{k=1}^{L} h_{i}^{2}(k)} \sqrt{\sqrt{\sum_{k=1}^{L} h_{j}^{2}(k)}}}
$$

Where $<\bullet>$ denotes inner production operator and $\|\bullet\|$ represents norm operator for a vector.

(e). Determination of two matched minutia pairs from two matched bars:

$$
\begin{cases}p_{i}^{F} \leftrightarrow p_{j}^{G}, q_{i}^{F} \leftrightarrow q_{j}^{G} & \text { if }\left|u_{i}^{F}-u_{j}^{G}\right| \leq u_{0} \text { and }\left|v_{i}^{F}-v_{j}^{G}\right| \leq v_{0} \\ p_{i}^{F} \leftrightarrow q_{j}^{G}, q_{i}^{F} \leftrightarrow p_{j}^{G} & \text { if }\left|u_{i}^{F}-v_{j}^{G}\right| \leq u_{0} \text { and }\left|v_{i}^{F}-u_{j}^{G}\right| \leq v_{0}\end{cases}
$$

Minutia pairs satisfying all above conditions may be selected as candidate reference minutia pairs. Through rigid constraints of features derived from local geometric and texture information, matched reference minutia pairs could dramatically decrease. 


\section{Fingerprint alignment and global matching}

Jain[9] demonstrates minutia matching method utilizing polar coordinate system which is scale and rotation invariant. Suppose minutia $M_{i}^{F}$ in input fingerprint and minutia $M_{j}^{G}$ in template fingerprint are matched reference minutia, and alignment of input fingerprint and template fingerprint is carried out with locomotion and rotation of input fingerprint. Rotate angle of input fingerprint is $\theta^{r}=\alpha_{j}^{G}-\alpha_{i}^{F}$, and the coordinate vector of reference minutia is $\left(x^{r}, y^{r}\right)$. Then, minutia coordinate $\left(x_{i}, y_{j}\right)$ in Euclidean space can be transformed in to polar coordinate vector as follows:

$$
\left(\begin{array}{l}
r_{i} \\
e_{i} \\
\theta_{i}
\end{array}\right)=\left(\begin{array}{c}
\sqrt{\left(x_{i}-x^{r}\right)^{2}+\left(y_{i}-y^{r}\right)^{2}} \\
\arctan \left(\frac{y_{i}-y^{r}}{x_{i}-x^{r}}\right) \\
e_{i}+\theta^{r}
\end{array}\right)
$$

Where $\left(r_{i}, \theta_{i}\right)$ is the final polar coordinate vector.

In order to search matched minutia pairs effectively and to guarantee robust to fingerprint deformation, we propose a changeable sized boundary box of unique area in this paper, because unique area of boundary box allows consistent error tolerance to each minutia. In Euclidean coordinate system, unique area of boundary box can easily be obtained; however, in polar coordinate system, parameters of a boundary box can be determined as follows:

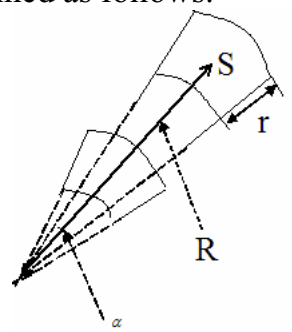

Fig. 4 Demonstration of changeable sized boundary box of unique area

In Figure 4, area of boundary box $S$ is fixed in the experiment, and radius of boundary box $r$ is also fixed in the experiment, then the polar angle of boundary box will decrease when polar radius of the corresponding minutia increases. The polar angle of boundary box can be calculated as follows:

$$
\alpha \approx S /(r * R)
$$

Where, all parameters in formula (11) are showed in Figure 5.

In order to determine whether two fingerprints are from the same source, we should calculate similarity of all possible minutia pairs in two fingerprints after alignment and compute global similarity of two fingerprints. Conditions that should be satisfied for two matched fingerprints are listed as follows:
a) $\left|\rho_{i}^{F}-\rho_{j}^{G}\right| \leq r / 2$
c) $\left|u_{i}^{F}-u_{j}^{G}\right| \leq u_{0}$
b) $\left|\alpha_{i}^{F}-\alpha_{j}^{G}\right| \leq \alpha / 2$
d) $s\left(M_{i}^{F}, M_{j}^{G}\right) \geq s_{0}$ 
Where, (a) and (b) are used to test whether two minutia are in the same boundary box or not; (c) is used to test whether two tangent directions of corresponding ridges are consistent; (d) is used to test texture similarity of two minutia regions. Statistic all matched minutia pairs between input and template fingerprints which is marked as $N_{\text {match }}$, and global similarity of two fingerprint can be estimated as follows:

$$
r_{\text {match }}=\frac{N_{\text {match }}}{\min \left\{N_{\text {input }}, N_{\text {template }}\right\}}
$$

Where, $r_{\text {match }}$ in formula (12) is regarded as minutia matching rate; $N_{\text {input }}$ is considered as number of valid minutia in input fingerprint; $N_{\text {template }}$ is deemed as number of valid minutia in template fingerprint. Eventually, we could judge two fingerprints are matched if $r_{\text {match }}$ is larger than a fixed threshold, and a optimal threshold should be determined in experiments in order to get global optimization to all performance indicators which will be discussed in section 5 .

\section{Experimental results}

\subsection{Advantages of gradient angular histogram as texture feature}

In this experiment, we choose fingerprint images 1_3.gif and 1_6.gif representing average quality fingerprint and low quality fingerprint respectively from sub-database DB1_A in FVC 2004.

During the process of searching matched bi-minutia bars in this experiment, parameters involved are defined as follows: (1) Euclidean length of bi-minutia bar is confined in range between 5 and 15 times of ridge period, namely [10 80]; (2) Error constraint of bi-minutia bar's Euclidean length $d_{0}=4$; (3) Error constraint of ridges that bi-minutia bar passes through $n_{0}=1$; (4) Directional deviation constraint between ridge and bi-minutia bar of certain minutia $u_{0}=v_{0}=\pi / 12 ;(5)$ Texture similarity constraint of gradient angular histogram $s_{0}=0.90$;

Table 1. Comparison of candidate reference minutia pairs

\begin{tabular}{llccl}
\hline Fingerprint & $\begin{array}{l}\text { Minutia num- } \\
\text { bers }\end{array}$ & $\begin{array}{c}\text { Matched bars } \\
\text { Bi-minutia bars (without considering gradi- } \\
\text { ent angular histogram) }\end{array}$ & $\begin{array}{c}\text { Matched bars } \\
\text { (after utilizing gradi- } \\
\text { ent angular } \\
\text { histogram) }\end{array}$ \\
\hline 1_3.gif & 62 & 421 & 565 & 57 \\
1_6.gif & 37 & 221 & 565 & 57 \\
\hline
\end{tabular}

From TABLE 1, we can observe that candidate reference bi-minutia bars decrease from 565 to 57 after considering constraint of texture similarity of gradient 
angular histogram, so that the amount of calculation of subsequent global matching will reduce about 9-10 times. Eventually, the ratio of retained candidate reference bi-minutia bars to all involved bars is $\frac{57}{421 \times 221}=0.06 \%$.

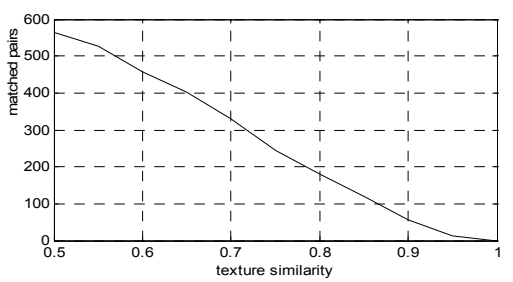

Fig. 5. Curve in the graph represents the number of reference minutia pairs with similarity of gradient angular histogram of images 1_3.gif and 1_6.gif in DB1_A of FVC2004.

From Figure 5, we can observe that matched candidate bi-minutia bars will decrease dramatically when texture similarity as constraint becomes higher. However, the threshold of texture similarity shouldn't be too high from numerous experimental observations. If the threshold of texture similarity is too high, it will omit many genuine and vital bi-minutia bars; inversely, matched bi-minutia bars will increase significantly.

After selecting optimal candidate reference bi-minutia bar, parameters used to statistic global matched minutia are defined as follows: (1) Radius error of boundary box $r=10$; (2) Polar angular error of boundary box $\alpha=S /(r \times R)=10 / R$; (3) Tangent directional error of two corresponding ridges $u_{0}=\pi / 12$; (4) Threshold of texture similarity constraint $s_{0}=0.9$.

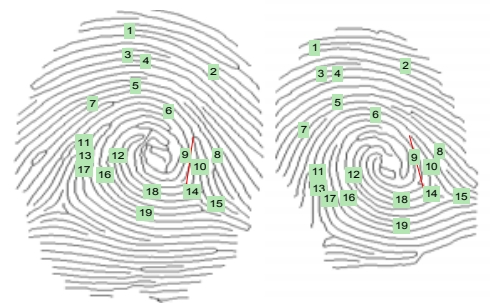

Fig. 6 Matched minutia pairs in two images of the same fingerprint; the red lines in the picture denotes optimal reference bi-minutia bar; the blue numbers in the picture represent corresponding orders of matched minutia pairs. The left is the ridge image of 1_3.gif in DB1_A of FVC2004, while the right is 1_6.gif.

From Figure 6, we can observe that the algorithm proposed in this paper can accurately find reference bi-minutia bar and obtain matched minutia pairs of the whole fingerprint. In this case, it has detected 19 pairs of matched minutia, and smaller minutia set has 37 valid minutia points, thus final minutia matching rate attain 50\%. 


\subsection{Performance of our method on FVC2004}

Nowadays, performance indicators of fingerprint matching that are wildly accepted include FNMR, FMR, EER, FNMR100, FNMR1000 and ZeroFMR. All above indicators can be reflected from the ROC curve, whose horizontal axis denotes FMR and vertical axis denotes FMNR.

Every subset in FVC2004 contains 100 fingerprints, and each fingerprint has 8 samples, thus it has 800 fingerprint images in a subset. Experimental data in estimating FNMR has $((8 * 7) / 2) * 100=2,800$ pairs; and total Experimental data in estimating FMR has $((100 * 99) / 2)=4,950$ pairs when only utilizing the first sample for each fingerprint.

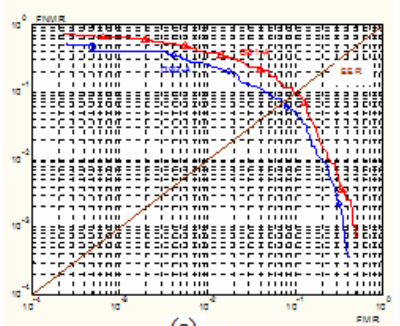

(a)

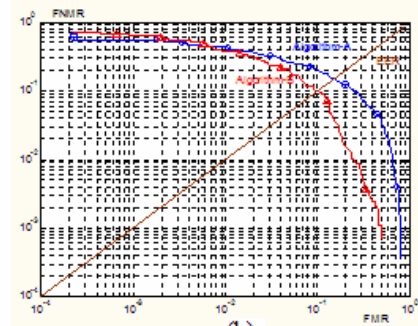

(b)

Fig. 7 ROC curves of the experiment results for database FVC2004. (a) Red curve illustrates the experiment results of DB1_A of FVC2004, whilst blue demonstrates DB2_A. (b) Blue curve represents the experiment result of bi-minutia bar model without texture similarity for DB1_A in FVC2004, whilst red represents the experiment result of our method for DB1_A.

From Figure 7(b), we can observe that ROC curve of our method in this paper illustrates better performance than that of method only considering bi-minutia bar model. Moreover, EER is about $10 \%$ observed from EER line, which is much lower than method only utilizing bi-minutia bar model whose EER is $\mathbf{1 5 \%}$.

Table 2. Results of Our New Method over the Two Databases among FVC2004

\begin{tabular}{lllll}
\hline $\begin{array}{l}\text { Database } \\
\text { (FVC2004) }\end{array}$ & $\begin{array}{l}\text { EER } \\
(\%)\end{array}$ & $\begin{array}{l}\text { FMR100 } \\
(\%)\end{array}$ & $\begin{array}{l}\text { FMR1000 } \\
(\%)\end{array}$ & $\begin{array}{l}\text { ZeroFMR } \\
(\%)\end{array}$ \\
\hline DB1_A & 9.56 & 17.2 & 23.0 & 37.2 \\
DB2_A & 7.46 & 20.0 & 25.4 & 42.0 \\
\hline
\end{tabular}

Observing from TABLE 2, experimental results of the algorithm in this paper are actually close with that of many excellent algorithms [10],[11], which demonstrates robustness and reliance of our method in this paper. Of course, the experimental results will become much better if we have a good performance of fingerprint preprocessing, such as segmentation, enhancement and binarization. Especially, many genuine minutia points will be omitted and pseudo minutia points will be forged if parameters of Gabor filter are not optimal. 


\section{Conclusions}

In this paper, we introduce a novel algorithm of fingerprint matching based on combination of minutia geometric and texture features. We adopt gradient angular histogram as texture feature in this paper, which effectively represents fingerprint information where minutia locate, because it can generally reflect profile of ridge edges and minutia types to some extent. In addition, we adopt bi-minutia bar model [10] as the geometric feature in this paper.

The new texture feature of gradient angular histogram in this paper can guarantee the accuracy of minutia matching of fingerprint; nonetheless, the gradient angular histogram will create deviation for the reason of low quality fingerprint. In the process of global fingerprint matching, using ratio of matched minutia pairs to total minutia can largely measure the similarity of two fingerprints. However, the matching accurateness might be improved if we could measure similarities from diverse aspects of corresponding weights [8].

\section{References}

[1] Lin Hong, Fingeprint Image Enhancement: Algorithm and Performance Evaluation, IEEE Trans. Pattern Analysis and Machine Intelligence, Vol. 20, No. 8, August 1998.

[2] Zhu En, Automatic fingerprint recognition technology, Publishing House of National University of Technology of Security, May 2006:95-107.

[3] Xiping Luo, Knowledge Based Fingerprint Image Enhancement, 15th ICPR, Vol.4, P783-786.

[4] Jie Tian, Technology of Biometric Feature Recognition and its Application, Publishing House of Electronic Industry, September 2005: 85-97.

[5] Rafael C. Gonzalez, Digital Image Processing (Second Edition), Publishing House of Electronics Industry, July 2005:567-585.

[6] Xiping Luo, A minutia matching algorithm in fingerprint verification, 15th ICPR, Vol.4, pp.833 836, Barcelona, 2000.

[7] Xudong Jiang, Fingerprint minutiae matching based on the local and global structures, IEEE,2000:1042 1045.

[8] MiaoLi Wen, Integration of multiple fingerprint matching algorithms, Proceedings of the Fifth International Conference on Machine Learning and Cybernetics, Dalian, 13-16 August 2006.

[9] A.K.Jain,LinHong,On-line identity authentication system using fingerprints, Proceedings of IEEE, 1997,85:1365 1388.

[10] Yuliang He,Jie Tian, Fingerprint Matching Based on Global Comprehensive Simility, IEEE Trans. Pattern Analysis and Machine Intelligence, Vol. 28, No. 6, August 2006.

[11] Zhu En, Automatic fingerprint recognition technology, Publishing House of National University of Technology of Security, May 2006:138-154.

[12] D.Isenor, S.Zaky . Fingerprint identification using graph matching. Pattern Recognition, 1986,19:113 122

[13] XiaJian Chen,Jie Tian. A matching algorithm based on local topologic structure. Proceedings of ICIAR2004, LNCS3211,2004:360 367.

[14] Jain A.K.,Hong L..Filterbank-based Fingerprint matching. IEEE Transactions on Image Processing, 2000, 19(5):846 859.

[15] Aparecido Nilceu Marana. Ridge-Based Fingerprint Matching Using Hough Transform. Proceedings of the XVIII Brazilian SIBGRAPI'05:1530 1834. 